\title{
A New Approach for Edge Detection
}

\author{
T. Sankara Rao \\ Associate Professor, \\ GIT, GITAM University
}

\author{
Prasad Reddy P.V.G.D \\ Professor \\ Andhra University
}

\author{
G. Srinivas, $P h D$ \\ Assistant Professor, \\ GIT, GITAM University
}

\begin{abstract}
A problem of fundamental importance in image processing is edge detection since an edge characterizes the boundaries. Edge detection filters out useless data, noise and frequencies while preserving the important structural properties in an image for further analysis and implementation. Due to limitations of the existing techniques finding a better method for edge detection is still an active area of research.. In order to augment the high-frequency components of an image in this paper we propose a new class of filter by name 'PSS filter' which implies a spatial filter shape with a high positive component at the centre. It is found that sharpening with PSS filter high lights some of the fine details of an image and enhances the clarity of its boundaries. Since the perception of human of image quality is not adequate some image quality metrics like Mean Square Error (MSE), Peak Signal to Noise Ratio (PSNR), Average Difference (AD), Normalized Absolute Error (NAE), Structural Content (SC), Normalized Cross Correlation (NCC) and Maximum Difference (MD) were employed for measurement of image quality. Experimental results show that the proposed PSS filter displayed better performance and superior noise resilience.
\end{abstract}

\section{Keywords}

Image processing, spatial filter, image sharpening, image smoothing, PSS filter, image quality metrics

\section{INTRODUCTION}

An image may be defined as a two dimensional function, $f(x$, $\mathrm{y}$ ), where $\mathrm{x}$ and $\mathrm{y}$ are spatial (Plane) coordinates, and the amplitude at any pair of co-ordinates $(\mathrm{x}, \mathrm{y})$ is called the intensity or grey level of the image at that point.

Edge detection is a low level operation used in image processing and computer vision applications. The main goal of edge detection is to locate and identify sharp discontinuities from an image. These discontinuities are due to abrupt changes in pixel intensity which characterizes boundaries of objects in a scene. Edges give boundaries between different regions in the image. These boundaries are used to identify objects for segmentation and matching purpose .These object boundaries are the first step in many of computer vision algorithms like edge based face recognition, edge based obstacle detection, edge based target recognition, image compression etc.

So the edge detectors are required for extracting the edges. There are many edge detection operators available [2]. These operators identifying vertical, horizontal, corner and step edges. The quality of edges detected by these operators is highly dependent on noise, lighting conditions, objects of same intensities and the density of edges in the scene.

There are two ways through which image quality can be assessed, Subjective and Objective. The evaluation based on observers is called Subjective which are time consuming and less accurate. Whereas the subsequent method is objective method of testing the image quality based on mathematical calculations [9].

\section{SHARPENING FILTERS}

The important area in the field of computer vision is edge detection. Edges classify the boundaries flanked by regions in an image an edge is the boundary between an object and the background, and indicates the boundary between overlapping objects [6]. As the color and brightness values for each pixel are interpolated some image softening is applied to even out any fuzziness that has occurred. To preserve the impression of depth, clarity and fine details, the image processor must sharpen edges and contours. It therefore must detect edges correctly and reproduce them smoothly and without oversharpening.

Sobel, Prewitt and Laplacian filters are one among the sharpening filters where each point in the image are done convolution with these two kernels. One kernel has a maximum response to the usual vertical edges and the other kernel has a maximum response to the horizontal edge. It is estimated in 8 possible directions and convolution result of greatest magnitude indicates the greatest direction.

\section{PSS Filter}

PSS operators perform a 2-Dimensional spatial ascent measurement on an image. Typically it can be used to unearth the approximate absolute gradient magnitude in an input gray scale image at every point. This edge detector uses a pair of $3 \times 3$ convolution masks, one estimating the gradient in the $\mathrm{x}-$ direction (columns) and the other estimating the gradient in the $y$-direction (rows). The size of a convolution mask is typically much smaller than the actual image. The ensuing image is untrained by sliding mask over an area of the input image, changing that pixel's value and then shifts one pixel to the right and continues until it reaches the end of a row. It then starts at the beginning of the next row. The proposed operators are in the form of a non singular matrix whose determinant is equal to zero.

The Gx mask brightens the edges in the horizontal direction while the Gy mask highlights the edges in the vertical direction

After taking the magnitude of both, the resulting output detects edges in both directions. The value of the gradient of proposed operators (both in $\mathrm{X}$ and $\mathrm{Y}$-directions) is positive so it identifies the boundaries clearly.

\begin{tabular}{|c|c|c|}
\hline-1 & -1 & -2 \\
\hline-1 & 12 & -2 \\
\hline-1 & -2 & -2 \\
\hline
\end{tabular}

\begin{tabular}{|c|c|c|}
\hline-1 & -1 & -1 \\
\hline-1 & 12 & -2 \\
\hline-2 & -2 & -2 \\
\hline
\end{tabular}


$\mathrm{Gx}=(-(\mathrm{Z} 1+\mathrm{Z} 2+\mathrm{Z} 4+\mathrm{Z} 7)-2(\mathrm{Z} 3+\mathrm{Z} 6+\mathrm{Z} 8+\mathrm{Z} 9)+12 \mathrm{Z} 5)$

$\mathrm{Gy}=(-(Z 1+Z 2+Z 3+Z 4)-(Z 6+Z 7+Z 8+Z 9)+12 Z 5)$

The above formula shows the way value of a particular pixel in the output image is calculated. The centre of the mask is placed over the pixel to be manipulated in the image.

\section{PSS FILTER OF $3 * 3$}

In the present case we use simplest approximations to a first derivative that satisfy the condition in equation [1] is used and the appropriate filter mask may be formulated as

$$
\begin{aligned}
& \nabla f \approx|12 Z 5|-|-(z 1+z 2+z 4+z 7)-2(Z 3+Z 6+Z 8+Z 9)|+ \\
& |12 z 5-(z 1+Z 2+Z 3+Z 4)-(Z 6+Z 7+Z 8+Z 9)+z 3|- \\
& |z 7+2 z 9| \rightarrow 1
\end{aligned}
$$

The above equation can be implemented with the two masks and these are referred as PSS - gradient operators.Generalized formula is used to generalize the given filter, but the filter can be generalized only by generalizing the equation i.e in this case it is equation [1]. Later one may increase or decrease the filter size according to quality of the image.

In a situation where one has to apply $3 * 3$ filter, then it is needed to divide the image into $3 * 3$ matrixes and then $3 * 3$ PSS mask has to slide over an area of the input image. Changing that pixel's value, and shifting one pixel to the right. It will then continue to the right and moves till the end of a row is reached. It then starts at the beginning of the next row.

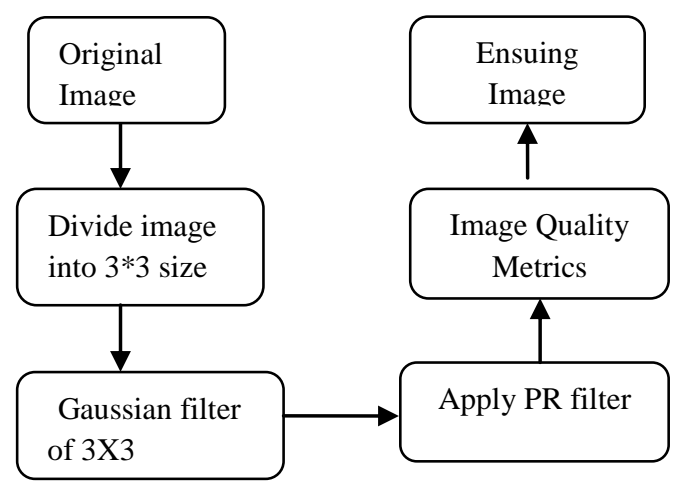

Figure 1: The Process of evaluation of PSS filter.

\section{Algorithm-I}

Step1: Read the Image.

Step2: partition the image into $3 * 3$ sizes.

Step3: Finding the edges of the image.

Work out the derivatives $G_{X}$ and $G_{Y}$ in $x$ and y directions. This can be done by the application of PSS filter which is of $3 * 3$ sizes for finding the edges of the image.

Step4: Smoothening the edges

Apply Gaussian filter of $3 \mathrm{X} 3$ size with sigma equal to 1.4 for smoothening the image.

\section{EXPERIMENTAL RESULTS}

This section presents the simulation results illustrating the performance of the proposed PSS filter. The fundamental significance in image processing is the measurement of image quality. In many image processing applications, assessment is required for image quality. The perception of human of image quality is not adequate. So we require some more image quality metrics like Mean Square Error (MSE), Peak Signal to Noise Ratio (PSNR), Average Difference (AD), Normalized Absolute Error (NAE), Structural Content (SC) and Maximum Difference (MD), Normalized Cross Correlation (NCC) and Maximum Difference for efficient measurement of image

\begin{tabular}{|c|c|}
\hline $\begin{array}{l}\text { Image Quality } \\
\text { Metric }\end{array}$ & Procedure to Calculate \\
\hline $\begin{array}{l}\text { Average } \\
\text { Difference }\end{array}$ & $\begin{array}{l}(\mathbf{1} \div \mathbf{M N}) \sum_{\mathrm{J}=\mathbf{1}}^{\mathbf{M}} \sum_{\mathbf{k}=\mathbf{1}}^{\mathbf{N}}\left[\mathbf{F}(\mathbf{X}, \mathbf{Y})-\mathbf{F}^{\prime}(\mathbf{X}, \mathbf{Y})\right] \\
\text { Where } \mathrm{M} \text { and } \mathrm{N} \text { are image rows and } \\
\text { columns in spatial form }\end{array}$ \\
\hline $\begin{array}{l}\text { Peak-Signal to } \\
\text { Noise Ratio }\end{array}$ & $\begin{array}{l}20 \log _{10}(255 / \text { MSE }) \\
\text { Where MSE is Mean Square Error }\end{array}$ \\
\hline $\begin{array}{l}\text { Mean Square } \\
\text { Error }\end{array}$ & $\begin{array}{l}(\mathbf{1} \div \mathbf{M N}) \sum_{\mathrm{J}=\mathbf{1}}^{\mathbf{M}} \sum_{\mathbf{k}=\mathbf{1}}^{\mathbf{N}}\left[\mathbf{F}(\mathbf{X}, \mathbf{Y})-\mathbf{F}^{\prime}(\mathbf{X}, \mathbf{Y})\right]^{2} \\
\text { Where } \mathrm{M} \text { and } \mathrm{N} \text { are image rows and } \\
\text { columns in spatial form }\end{array}$ \\
\hline $\begin{array}{l}\text { Maximum } \\
\text { Distance }\end{array}$ & $\mathbf{M A X}\left\{\left|\mathbf{F}(\mathbf{X}, \mathbf{Y})-\mathbf{F}^{\prime}(\mathbf{X}, \mathbf{Y})\right|\right\}$ \\
\hline $\begin{array}{l}\text { Structural } \\
\text { Content }\end{array}$ & $\begin{array}{l}\left.\sum_{\mathbf{J}=\mathbf{1}}^{\mathbf{M}} \sum_{\mathbf{k}=\mathbf{1}}^{\mathbf{N}}(\mathbf{F ( X , Y )})^{2} \div \sum_{\mathbf{J}=\mathbf{1}}^{\mathbf{M}} \sum_{\mathbf{k}=\mathbf{1}}^{\mathbf{N}}\left({ }_{\mathbf{F}}, \mathbf{X}, \mathbf{Y}\right)\right)^{2} \\
\text { Where } \mathrm{M} \text { and } \mathrm{N} \text { are image rows and } \\
\text { columns in spatial form }\end{array}$ \\
\hline $\begin{array}{l}\text { Normalized } \\
\text { Absolute Error }\end{array}$ & $\begin{array}{l}\left.\sum_{\mathbf{J}=\mathbf{1}}^{\mathbf{M}} \sum_{\mathbf{k}=\mathbf{1}}^{\mathbf{N}}\right|_{\left[\mathbf{F}(\mathbf{X}, \mathbf{Y})-\mathbf{F}^{\prime}(\mathbf{X}, \mathbf{Y})\right]}\left|\sum^{\mathbf{M}} \sum_{\mathbf{J}=\mathbf{1}}^{\mathbf{N}}\right|_{\mathbf{F}(\mathbf{X}, \mathbf{1})} \mid \\
\text { Where } \mathrm{M} \text { and } \mathrm{N} \text { are image rows and } \\
\text { columns in spatial form }\end{array}$ \\
\hline $\begin{array}{l}\text { Normalized } \\
\text { Cross } \\
\text { Correlation }\end{array}$ & $\begin{array}{l}\left.\left.\sum_{\mathbf{J}=\mathbf{1}}^{\mathbf{M}} \sum_{\mathbf{k}=\mathbf{1}}^{\mathbf{N}}\right|_{\left[\mathbf{F}(\mathbf{X}, \mathbf{Y})^{*} \mathbf{F}^{\prime}(\mathbf{X}, \mathbf{Y})\right]}\left|\sum^{\mathbf{M}} \sum_{\mathbf{J}=\mathbf{1}}^{\mathbf{N}}\right|_{\mathbf{k}=\mathbf{1}}(\mathbf{X}, \mathbf{Y})\right|^{2} \\
\text { Where } \mathrm{M} \text { and } \mathrm{N} \text { are image rows and } \\
\text { columns in spatial form }\end{array}$ \\
\hline Fidelity criteria & 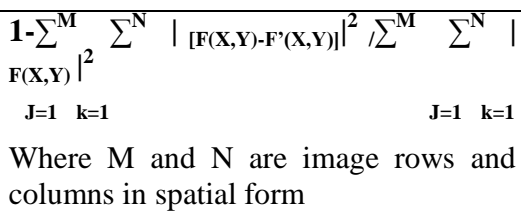 \\
\hline
\end{tabular}
quality

Image quality metric Formulae

\begin{tabular}{|l|l|l|}
\hline 0.0924 & 0.1192 & 0.0924 \\
\hline 0.1192 & 0.1538 & 0.1192 \\
\hline 0.0924 & 0.1192 & 0.0924 \\
\hline
\end{tabular}




\begin{tabular}{|l|l|}
\hline $\begin{array}{l}\text { Mean Square } \\
\text { Error }\end{array}$ & $\begin{array}{l}(\mathbf{1} \div \mathbf{M N}) \sum_{\mathbf{J}=\mathbf{1}}^{\mathrm{M}} \sum_{\mathbf{k}=\mathbf{1}}^{\mathrm{N}}\left[\mathbf{F}(\mathbf{X}, \mathbf{Y})-\mathbf{F}^{\prime}(\mathbf{X}, \mathbf{Y})\right]^{2} \\
\text { Where } \mathrm{M} \text { and } \mathrm{N} \text { are image rows and } \\
\text { columns in spatial form }\end{array}$ \\
\hline
\end{tabular}

\subsection{Tabular values of quality metrics for each filter}

Table 1: Comparison of Image Quality Metrics for PSS Filter.

\begin{tabular}{|c|c|c|c|c|c|c|c|c|c|}
\hline Sno & Image & PSNR & MSE & NAE & AD & MD & SC & RMSE & NCC \\
\hline 1 & Calculator.tif & 11.12 & 0.501 & 0.81 & 58.04 & 255 & 3.60 & 70.82 & 0.30 \\
\hline 2 & Building_original & 5.21 & 1.95 & 0.84 & 105.19 & 255 & 7.18 & 139.93 & 0.16 \\
\hline 3 & Skull.tif & 10.94 & 0.523 & 0.864 & 28.28 & 243 & 7.83 & 72.35 & 0.158 \\
\hline 4 & Brain.tif & 5.804 & 1.708 & 0.95 & 121.33 & 226 & 22.89 & 130.70 & 0.062 \\
\hline 5 & Brain.thumb.tif & 11.552 & 0.454 & 0.821 & 16.875 & 144 & 0.97 & 67.439 & 0.666 \\
\hline 6 & Pills.tif & 5.136 & 1.992 & 0.902 & 130.24 & 214 & 15.64 & 141.16 & 0.108 \\
\hline 7 & Region.tif & 4.861 & 2.123 & 0.924 & 83.258 & 255 & 13.244 & 145.70 & 0.075 \\
\hline 8 & Rose1024.tif & 11.214 & 0.491 & 0.837 & 31.886 & 255 & 6.244 & 70.11 & 0.205 \\
\hline 9 & Tungsten_flmt.tiff & 7.953 & 1.041 & 0.845 & 82.263 & 253 & 6.247 & 102.063 & 0.12 \\
\hline 10 & u.tif & 7.295 & 1.212 & 0.947 & 47.53 & 255 & 19.099 & 110.092 & 0.052 \\
\hline 11 & Utk.tif & 19.384 & 0.749 & 0.619 & 2.824 & 255 & 2.139 & 27.374 & 0.436 \\
\hline 12 & Turbine.tif & 6.814 & 1.354 & 0.743 & 88.134 & 255 & 3.247 & 116.36 & 0.333 \\
\hline 13 & Cholesterol.tif & 9.680 & 0.699 & 0.752 & 25.153 & 251 & 1.004 & 83.66 & 0.698 \\
\hline 14 & Cameraman.tif & 6.661 & 1.402 & 0.852 & 84.897 & 248 & 3.025 & 118.42 & 0.275 \\
\hline
\end{tabular}

Table 2: Comparison of Image Quality Metrics for Sobel Filter

\begin{tabular}{|c|c|c|c|c|c|c|c|c|c|}
\hline Sno & Image & PSNR & MSE & NAE & $\mathrm{AD}$ & MD & $\mathrm{SC}$ & RMSE & $\mathrm{NCC}$ \\
\hline 1 & Calculator.tif & 10.13 & 0.631 & 0.8964 & 62.01 & 255 & 4.64 & 79.43 & 0.1848 \\
\hline 2 & Building_original & 4.84 & 2.13 & 0.8954 & 111.00 & 255 & 8.79 & 146.04 & 0.1170 \\
\hline 3 & Skull.tif & 10.62 & 0.562 & 0.90 & 29.04 & 243 & 10.74 & 75.02 & 0.111 \\
\hline 4 & Brain.tif & 5.76 & 1.7248 & 0.949 & 121.69 & 228 & 36.84 & 131.33 & 0.0493 \\
\hline 5 & Brain.thumb.tif & 9.92 & 0.66 & 1.008 & 32.574 & 239 & 2.086 & 81.307 & 0.2329 \\
\hline 6 & Pills.tif & 4.907 & 2.100 & 0.925 & 132.29 & 250 & 16.868 & 144.93 & 0.0831 \\
\hline 7 & Region.tif & 4.528 & 2.3 & 0.998 & 84.428 & 255 & 15.99 & 151.40 & 0.0322 \\
\hline 8 & Rose1024.tif & 10.6 & 0.566 & 0.89 & 40.678 & 255 & 22.502 & 75.256 & 0.0905 \\
\hline 9 & Tungsten_flmt.tiff & 7.52 & 1.15 & 0.89 & 85.644 & 255 & 8.26 & 107.26 & 0.14 \\
\hline 10 & u.tif & 7.04 & 1.28 & 1.003 & 48.680 & 255 & 33.978 & 113.313 & 0.0128 \\
\hline 11 & Utk.tif & 17.10 & 0.126 & 1.0217 & 3.645 & 255 & 3.299 & 35.594 & 0.1484 \\
\hline 12 & Turbine.tif & 5.68 & 1.755 & 0.892 & 118.92 & 255 & 23.39 & 132.50 & 0.1056 \\
\hline 13 & Cholesterol.tif & 7.99 & 1.031 & 0.9163 & 36.467 & 254 & 1.40 & 101.55 & 0.4156 \\
\hline 14 & Cameraman.tif & 6.0164 & 1.6272 & 0.9300 & 99.417 & 253 & 6.712 & 127.56 & 0.1220 \\
\hline \multicolumn{4}{|c|}{$\begin{array}{l}\text { The mean square error (MSE) is the measure of differenc } \\
\text { between actual and estimated value of the quantity]. Large } \\
\text { the value of MSE implies poor quality of the image. The mos } \\
\text { familiar image quality metric is PSNR. If PSNR value is hig } \\
\text { then the difference between the original image an } \\
\text { reconstructed image will be small. Large the value of SC }\end{array}$} & \multicolumn{6}{|c|}{$\begin{array}{l}\text { obtain an uncontaminated and less noisy image the value of } \\
\text { Average Difference (AD) should be reduced. The quality of } \\
\text { the image increases with decrease in the value of NAE, } \\
\text { Higher the value of NAE means the quality of the image } \\
\text { lower. NCC is the measure of calculating the degree of } \\
\text { resemblance between two objects. }\end{array}$} \\
\hline
\end{tabular}


Table 3: Comparison of Image Quality Metrics for Prewitt Filter

\begin{tabular}{|c|c|c|c|c|c|c|c|c|c|}
\hline Sno & Image & PSNR & MSE & NAE & AD & MD & SC & RMSE & NCC \\
\hline 1 & Calculator.tif & 10.06 & 0.64 & 0.911 & 64.64 & 255 & 6.04 & 80.04 & 0.1534 \\
\hline 2 & Building_original & 4.788 & 2.15 & 0.90 & 116.18 & 255 & 13.71 & 146.93 & 0.09 \\
\hline 3 & Skull.tif & 10.52 & 0.575 & 0.91 & 30.38 & 243 & 17.10 & 75.88 & 0.0839 \\
\hline 4 & Brain.tif & 5.70 & 1.75 & 0.96 & 123.49 & 228 & 53.69 & 132.31 & 0.0381 \\
\hline 5 & Brain.thumb.tif & 10.03 & 0.644 & 0.99 & 36.52 & 239 & 2.903 & 80.306 & 0.177 \\
\hline 6 & Pills.tif & 4.81 & 2.14 & 0.93 & 135.68 & 250 & 25.25 & 146.49 & 0.0636 \\
\hline 7 & Region.tif & 4.52 & 2.3 & 0.998 & 84.43 & 255 & 16.00 & 151.40 & 0.0322 \\
\hline 8 & Rose1024.tif & 10.45 & 0.585 & 0.9152 & 42.336 & 255 & 39.89 & 76.49 & 0.0665 \\
\hline 9 & Tungsten_flmt.tiff & 7.3893 & 1.18 & 0.9125 & 90.095 & 255 & 13.349 & 108.91 & 0.1045 \\
\hline 10 & u.tif & 7.045 & 1.28 & 1.003 & 48.680 & 255 & 33.978 & 113.313 & 0.0128 \\
\hline 11 & Utk.tif & 17.15 & 0.125 & 1.01 & 3.696 & 255 & 3.487 & 35.381 & 0.14 \\
\hline 12 & Turbine.tif & 5.49 & 1.83 & 0.92 & 123.15 & 255 & 43.53 & 135.51 & 0.076 \\
\hline 13 & Cholesterol.tif & 8.32 & 0.956 & 0.865 & 47.26 & 254 & 2.064 & 97.795 & 0.3327 \\
\hline 14 & Cameraman.tif & 5.997 & 1.64 & 0.9377 & 103.535 & 253 & 9.608 & 128.13 & 0.0955 \\
\hline
\end{tabular}

Table 4: Comparison of Image Quality Metrics for Laplacian Filter

\begin{tabular}{|c|c|c|c|c|c|c|c|c|c|}
\hline Sno & Image & PSNR & MSE & NAE & $\mathrm{AD}$ & MD & $\mathrm{SC}$ & RMSE & $\mathrm{NCC}$ \\
\hline 1 & Calculator.tif & 9.70 & 0.70 & 0.94 & 70.96 & 255 & 54.81 & 83.47 & 0.04 \\
\hline 2 & Building_original & 4.49 & 2.31 & 0.95 & 126.81 & 255 & 108.12 & 152.05 & 0.02 \\
\hline 3 & Skull.tif & 10.15 & 0.62 & 0.97 & 32.72 & 243 & 42.84 & 79.21 & 0.0263 \\
\hline 4 & Brain.tif & 5.50 & 1.83 & 0.99 & 128.03 & 229 & 0.186 & 135.28 & 0.0076 \\
\hline 5 & Brain.thumb.tif & 9.527 & 0.724 & 1.096 & 35.314 & 255 & 3.165 & 85.145 & 0.102 \\
\hline 6 & Pills.tif & 4.569 & 2.27 & 0.976 & 143.15 & 250 & 300.7 & 150.68 & 0.018 \\
\hline 7 & Region.tif & 4.323 & 2.402 & 1.05 & 85.476 & 255 & 21.59 & 155.01 & 0 \\
\hline 8 & Rose1024.tif & 10.331 & 0.602 & 0.922 & 42.769 & 255 & 95.188 & 77.615 & 0.046 \\
\hline 9 & Tungsten_flmt.tiff & 7.021 & 1.29 & 0.963 & 98.367 & 255 & 107.644 & 113.618 & 0.033 \\
\hline 10 & u.tif & 6.841 & 1.345 & 1.054 & 47.44 & 255 & 19.214 & 115.99 & 0 \\
\hline 11 & Utk.tif & 16.432 & 0.147 & 1.223 & 3.914 & 255 & 5.189 & 38.452 & 0.009 \\
\hline 12 & Turbine.tif & 5.469 & 1.845 & 0.910 & 121.29 & 255 & 34.354 & 135.84 & 0.077 \\
\hline 13 & Cholesterol.tif & 7.841 & 1.068 & 0.908 & 68.224 & 255 & 8.515 & 103.38 & 0.101 \\
\hline 14 & Cameraman.tif & 5.78 & 1.718 & 0.966 & 108.49 & 253 & 22.413 & 131.07 & 0.044 \\
\hline
\end{tabular}

\subsection{Graphical comparison of filters for each quality metric}

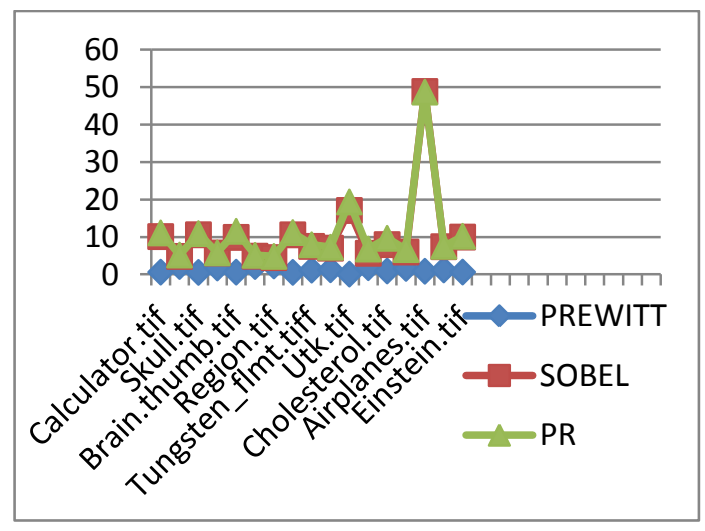

PSNR

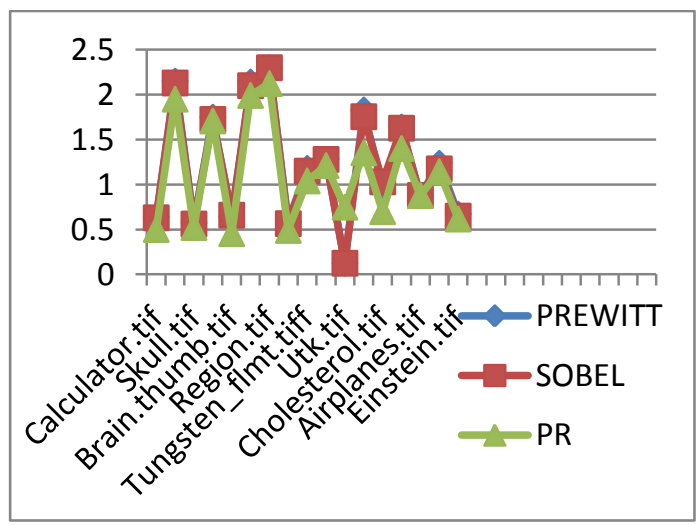

MSE 


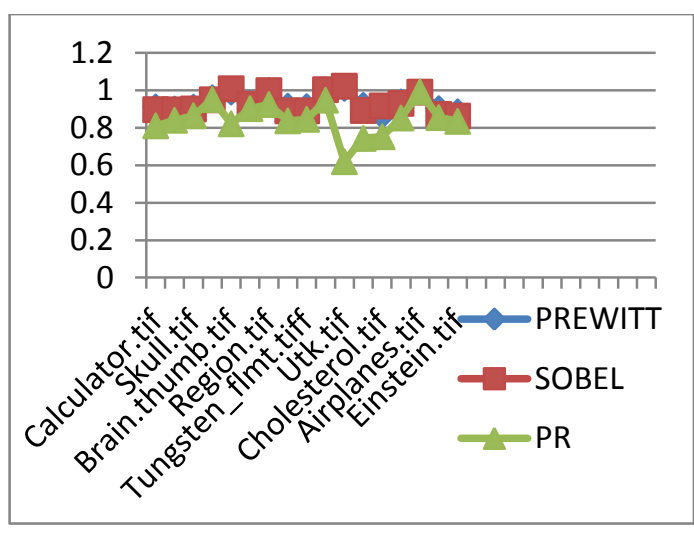

NAE

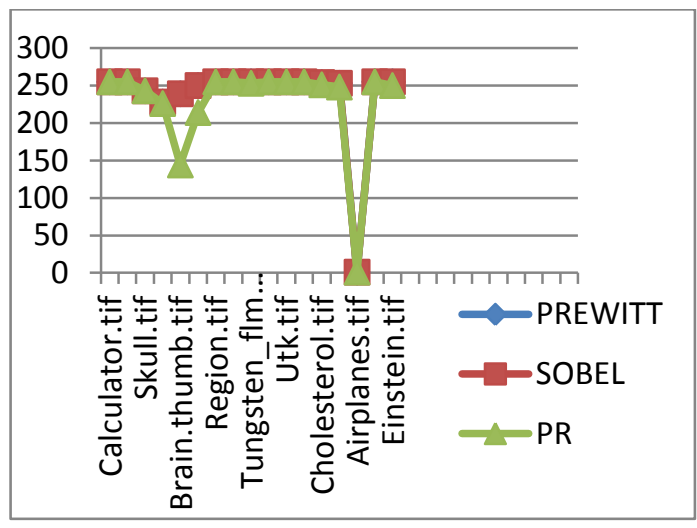

MD

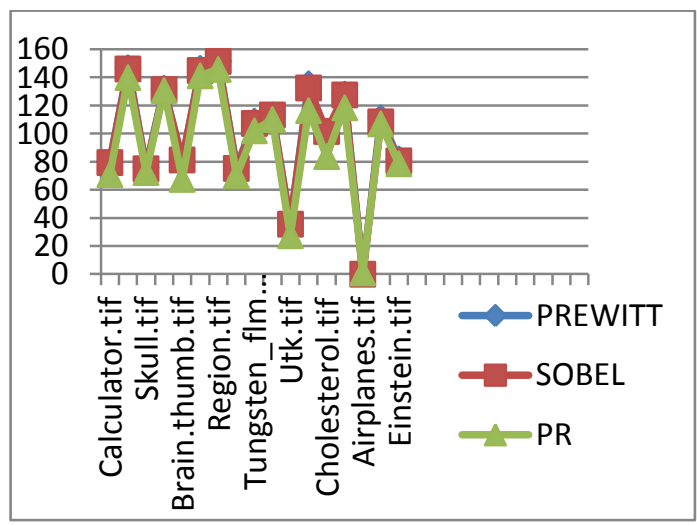

RMSE

\subsection{Visual comparison of filters}

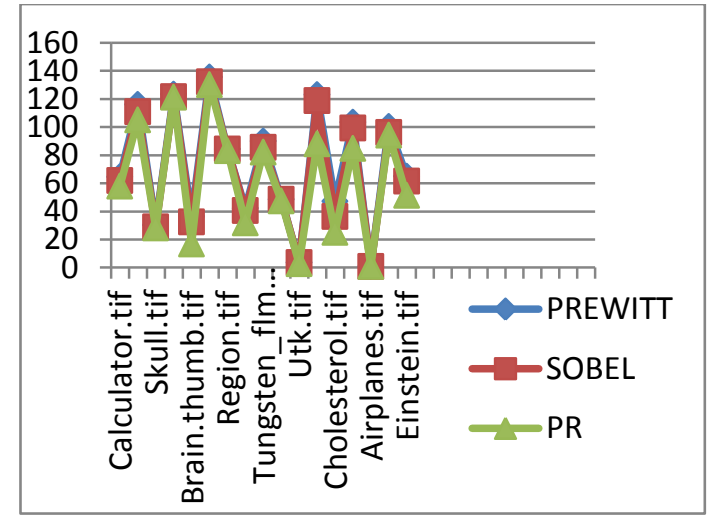

AD

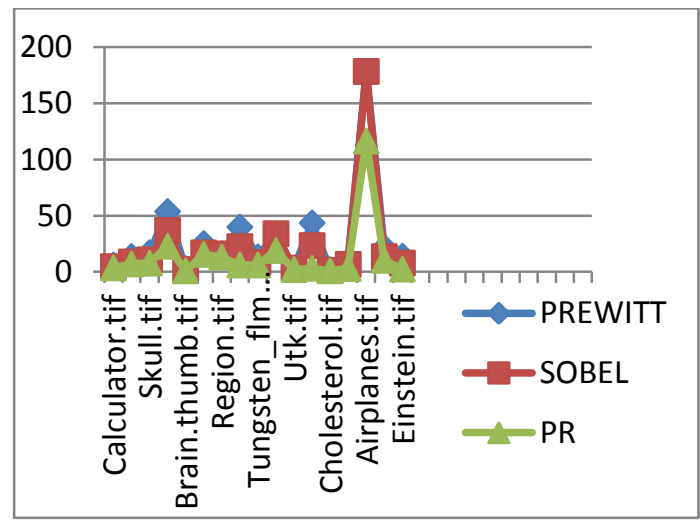

SC

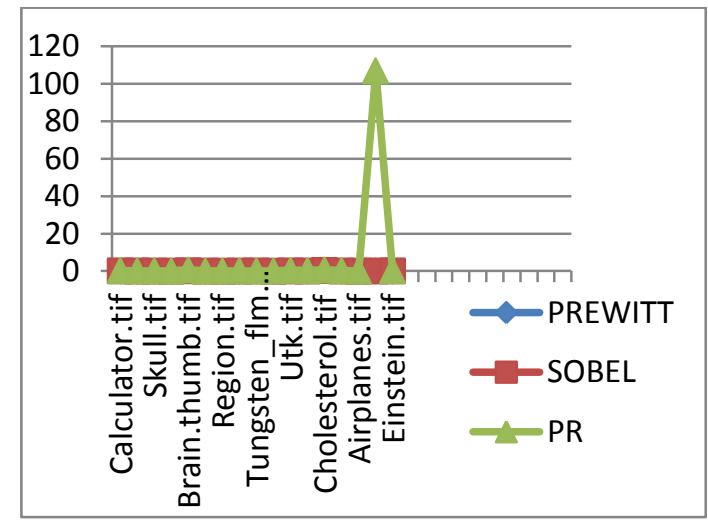

NAE 

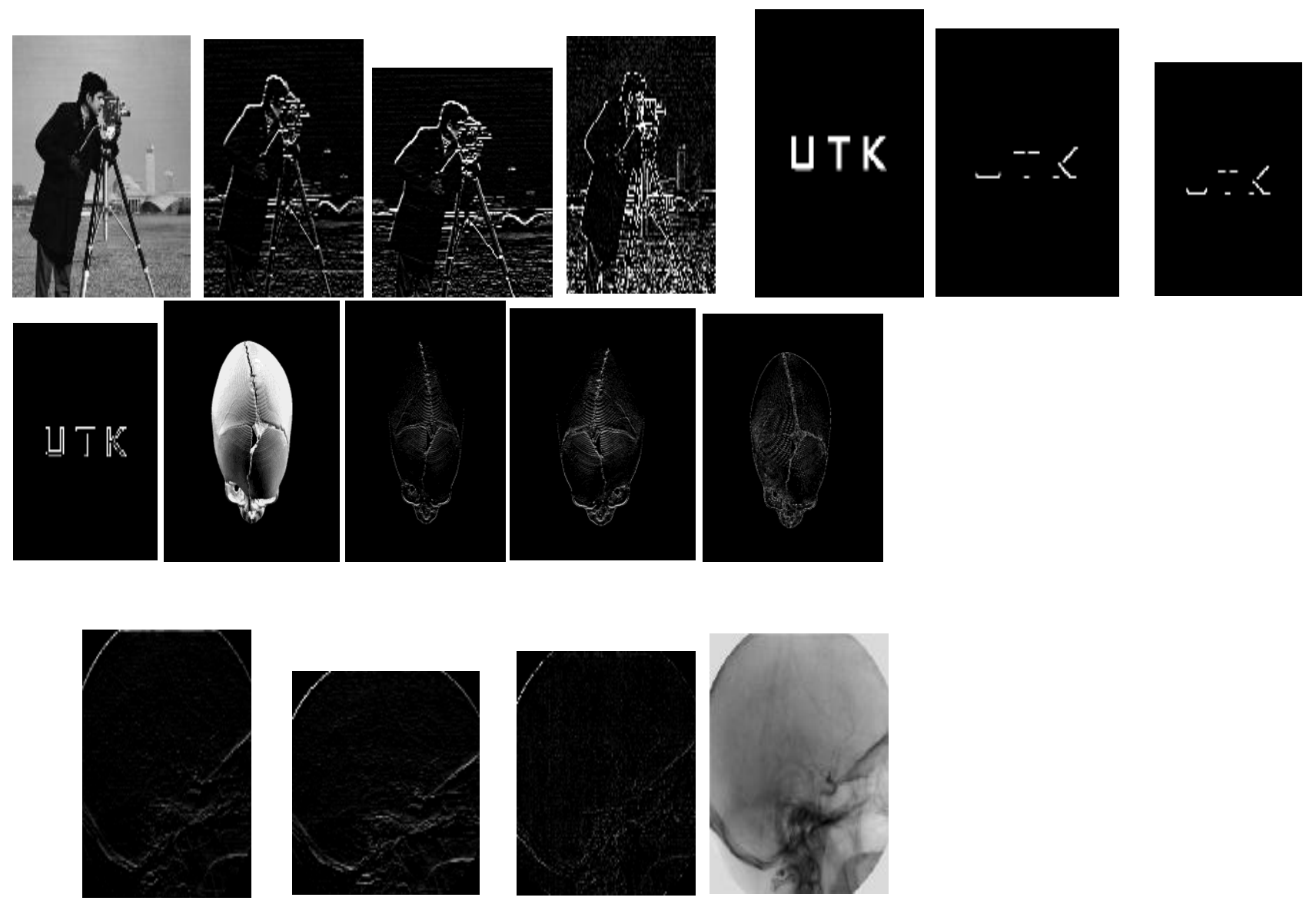

a) Original image

(b) Sharpened with Prewitt

(c) Sharpened with Sobel

(d) Sharpened with PSS filter

\subsection{Tabular comparison of filters}

Table 5: Tabular comparison of filters image wise

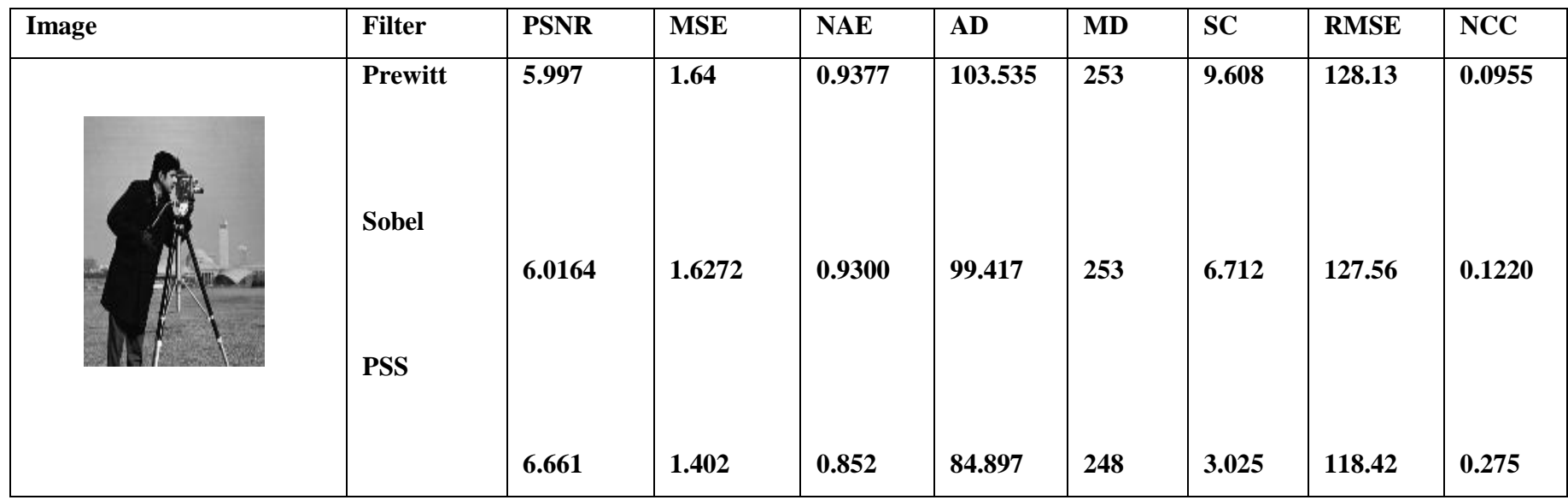




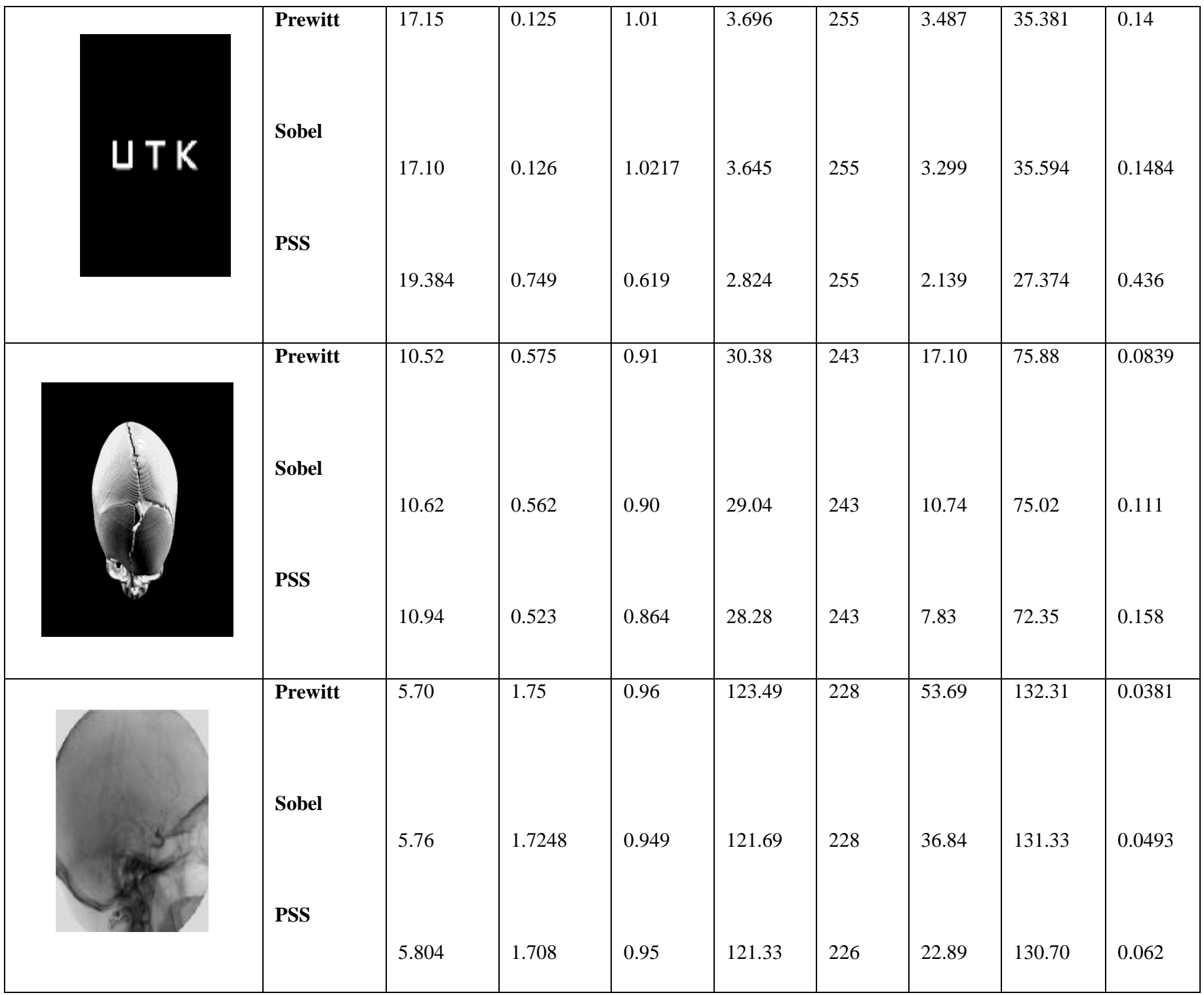

The above results of the work show that among the analyzed schemes the proposed PSS filter exhibits better results.

\section{CONCLUSION}

In this effort we proposed a new approach called PSS filter. It has wide applicability in many areas like satellite image enhancement, medical diagnostics, etc. The trialing and simulations with real images have shown that the filter is efficient than other existing filters. Several existing techniques for edge detection in image processing have been compared with PSS filter. Some of image processing metrics such as MSE, PSNR, AD, MD, SC, NAE, NCC are employed in this assessment. The code for these new filters has been generated and the obligatory strategies of comparisons between proposed and existing filters are tabulated. The values of MSE, SC, NAE, MD, AD are low and the values of PSNR and NCC are high for regular images are enhanced with PSS filter than Prewitt, Sobel and Laplacian operators, indicates the PSS filter identifies better edges than Prewitt, Sobel and Laplacian filters.

\section{REFERENCES}

[1] An Image-Enhancement System Based on Noise Estimation Fabrizio Russo, Senior Member, IEEE, IEEE

\section{TRANSACTIONS ON INSTRUMENTATION AND MEASUREMENT, VOL. 56, NO. 4, AUGUST 2007.}

[2] Gradient Estimation Using Wide Support Operators Hakan Guray Senel, Member, IEEE, IEEE TRANSACTIONS ON IMAGE PROCESSING, VOL. 18, NO. 4, APRIL 2009

[3] Gaussian-Based Edge-Detection Methods-A Survey Mitra Basu, Senior Member, IEEE, IEEE TRANSACTIONS ON SYSTEMS, MAN, AND CYBERNETICS-PART C: APPLICATIONS AND REVIEWS, VOL. 32, NO. 3, AUGUST 2002

[4] Gray-Scale Image Enhancement as an Automatic Process Driven by Evolution, Cristian Munteanu and Agostinho Rosa, IEEE TRANSACTIONS ON SYSTEMS, MAN, AND CYBERNETICS-PART B: CYBERNETICS, VOL. 34, NO. 2, APRIL 2004

[5] An Image-Enhancement System Based on Noise Estimation Fabrizio Russo, Senior Member, IEEE, TRANSACTIONS ON INSTRUMENTATION AND MEASUREMENT, VOL. 56, NO. 4, AUGUST 2007. 
[6] Edge Detection Techniques: Evaluations and Comparisons Ehsan Nadernejad, Applied Mathematical Sciences, Vol. 2, 2008, no. 31, $1507-1520$.

[7] A survey of image spamming and filtering techniques Abdolrahman Attar - Reza Moradi Rad ,Reza Ebrahimi Atani, (C) Springer Science+Business Media B.V. 2011.

[8] Zhou Wang, Member, IEEE, Guixing Wu, Hamid Rahim Sheikh,Member, IEEE, Eero P. Simoncelli, Senior Member, IEEE, En- Hui Yang, Senior Member, IEEE, and Alan Conrad Bovik, Fellow, IEEE, "Quality Aware Images", IEEE TRANSACTIONS ON IMAGE PROCESSING, VOL. 15, NO. 6, JUNE 2006.

[9] VQEG, "Final report from the video quality experts group on the validation of objective models of video quality assessment", Mar. 2000

[10] A Survey on Various Edge Detector TechniquesSaket Bhardwaja, Ajay Mittalb 2212-0173 (C) 2012 Published by Elsevier Ltd. 